\title{
Interferometric monitoring of dip coating
}

\author{
Alexandre F. Michels, Thiago Menegotto, and Flavio Horowitz
}

\begin{abstract}
Dip-coated films, which are widely used in the coating industry, are usually measured by capacitive methods with micrometric precision. For the first time to our knowledge, we have applied an interferometric determination of the evolution of thickness in real time to nonvolatile Newtonian mineral oils with several viscosities and distinct dip withdrawing speeds. The evolution of film thickness during the process depends on time as $t^{-1 / 2}$, in accordance with a simple model. Comparison with measured results with an uncertainty of $\pm 0.007 \mu \mathrm{m}$ ) showed good agreement after the initial steps of the process had been completed. () 2004 Optical Society of America
\end{abstract}

OCIS codes: $310.3840,310.6860,260.3160$.

\section{Introduction}

Continuous or batch-dip coating processes are used especially for nonplanar and long-dimensioned objects. ${ }^{1,2}$ The resultant film thicknesses are traditionally measured by capacitive techniques with typical uncertainties of $\pm 2 \mu \mathrm{m},{ }^{3}$ which are also influenced by other parameters, such as variations in substrate and belt thickness.

Optical monitoring has been used in solid thin films under vacuum deposition since the $1950 \mathrm{~s},{ }^{4}$ however; its application to spin coating of liquid films started four decades later.,, 6

An interferometric technique for dip coating was reported by Qu et al. ${ }^{7}$ that measures the film's spatial profile. Temporal evolution was the subject of a previous report in the feasibility of monitoring optical thickness in real time was shown. In that study a multicomponent zirconyl chloride aqueous solution was considered, and interpretation became difficult because of significant variations in refractive index during the dip-coating process. ${ }^{8}$

Aiming at a first comparison of experimental and theoretical results, here we chose to monitor Newtonian and nonvolatile liquids, which facilitate analysis with a simple model consisting of mineral oils with distinct properties.

The authors are with the Instituto de Física and Programa de Pós-Graduação em Ciências dos Materiais, Universidade Federal do Rio Grande do Sul, Campus do Vale CP15051, 91501-970 Porto Alegre, RS, Brazil. F. Horowitz's e-mail address is flavio@if.ufrgs. br.

Received 8 August 2003; accepted 6 November 2003.

0003-6935/04/040820-04\$15.00/0

(C) 2004 Optical Society of America

\section{Experiment}

In the experiment illustrated in Fig. 1(a), a laser beam at a wavelength of $\lambda=660 \mathrm{~nm}$ is measured after normal reflection by a liquid film flowing onto a glass substrate. As in optospinography, ,,6 the reflected light is compared with a reference signal and later processed by a lock-in amplifier and a computer with an analog-to-digital converter at a rate of 3.5 $\mathrm{kHz}$. The spot that is measured corresponds to a fixed value on the vertical $x$ axis.

This high acquisition rate and the proper alignment of the optical system, which lead to a small illuminated spot on the sample (diameter, $<0.5 \mathrm{~mm}$ ) at $x=x_{0}$ were carefully chosen for detailed monitoring of the dip-coating process.

\section{Theory}

Dip coating is a simple, old film-deposition technique, but understanding it might require the introduction of complex physical or chemical interactions. Under steady-state flow, the evolution of the thickness of a liquid film upon a substrate of infinite length (continuous process) can be described by a simplified form of the Navier-Stokes equation as obtained by Landau and Levich ${ }^{9,10}$ :

$$
\sigma \frac{\partial^{3} h}{\partial x^{3}}+\mu \frac{\partial^{2} v_{x}}{\partial y^{2}}+\rho g=0,
$$

with regard to the coordinate system shown in Fig. 1(b) and when major effects on a fluid volume element with vertical velocity $v_{x}$ as related to surface tension $\sigma$, gravity force $\rho g$, and viscosity $\mu$ are taken into consideration.

The first force in Eq. (1) is associated with the pressure gradient, $P_{\sigma}$, caused by surface tension of 


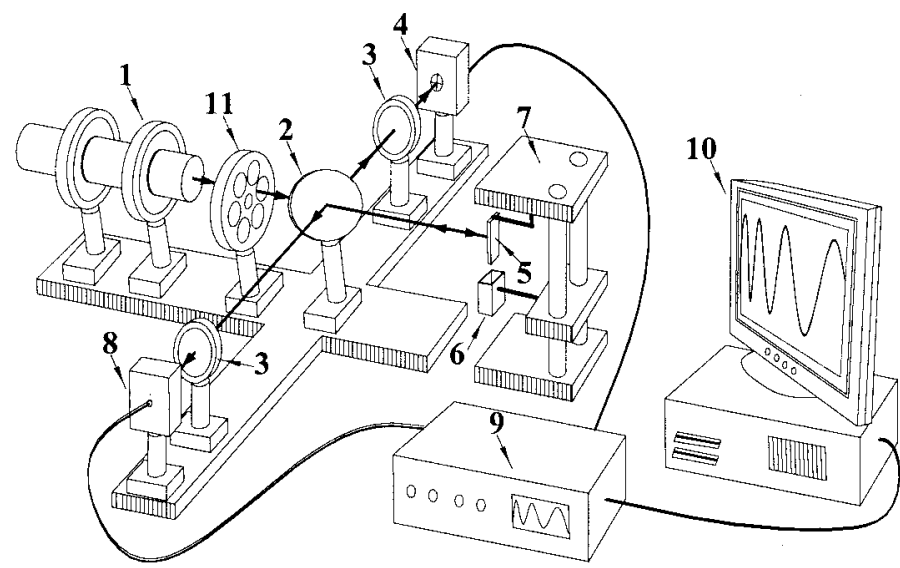

(a)

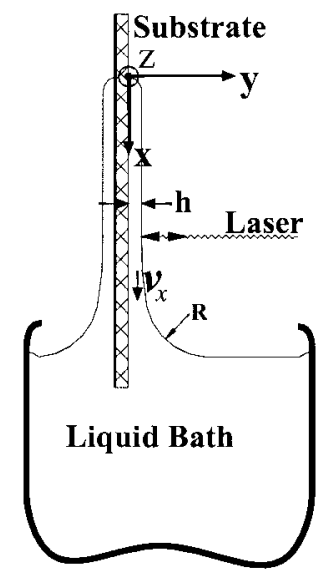

(b)

Fig. 1. (a) Illustration of the optical monitor, which comprises 1, a laser; 2, a beam splitter; 3, lenses; 4, a reference detector; 5 , a sample; 6, a liquid bath; 7, a dip-coater support; 8, a sample detector; 9, a lock-in amplifier; 10, a microcomputer; and 11, a chopper. (b) Schematic of the dip-coating batch process, where laser probing is shown.

the curved meniscus. When this effect is much smaller than those from the forces of gravity and viscosity (in connection with region 1 reported by Gutfinger and Tallmadge ${ }^{11}$ as well as by Spiers et $\left.a l .{ }^{3}\right)$, radius of curvature $R$ tends to infinity. Consequently,

$$
P_{\sigma}=-\sigma\left(\frac{\partial^{2} h}{\partial x^{2}}\right) \approx-\sigma\left(\frac{1}{R}\right) \approx 0
$$

and the force generated by surface tension can be neglected; that is, $\partial P_{\sigma} / \partial x \approx 0$. In this case Eq. (1) can be written as

$$
v \frac{\partial^{2} v_{x}}{\partial y^{2}}+g=0
$$

where $\mu / \rho=v$ is the kinematic viscosity.

For an observer at rest on the substrate, the boundary conditions for this system are

$$
v_{x}(y=0)=0,\left.\quad \frac{\partial v_{x}}{\partial y}\right|_{y=h}=0 .
$$

With these conditions, Eq. (3) leads to

$$
v_{x}(y)=\frac{g}{v}\left(h y-\frac{y^{2}}{2}\right) .
$$

Liquid flow $q$, taken as the average drainage velocity per film width unit (width in the $z$ direction), or

$$
q=\frac{1}{h_{0}} \int_{0}^{h_{0}} v_{x}(y) \mathrm{d} y,
$$

then becomes

$$
q=g \frac{h^{3}}{3 v}
$$

whereas the continuity equation for the noncompressible liquid is expressed as ${ }^{1}$

$$
\left(\frac{\partial h}{\partial t}\right)_{x}=-\left(\frac{\partial q}{\partial x}\right)_{t}=-\frac{\rho g h^{2}}{\mu} \frac{\partial h}{\partial x} .
$$

In the batch process, with a finite substrate such as shown in Fig. 1(b), if coincident origins of position and time are suitably chosen, the film thickness, as reported by Scriven, ${ }^{1}$ can be obtained from

$$
h=B \sqrt{\frac{\mu}{g \rho} \frac{x}{t}},
$$

where $x / t$ corresponds to the withdrawing speed for an observer in the laboratory reference system.

Under more-general conditions, in which the effect of the pressure gradient caused by the curved meniscus's surface tension plays a significant role (in connection with region 2 reported by Gutfinger and Tallmadge ${ }^{11}$ ), several theories indicate a relationship similar to Eq. (9) with a proportionality constant $C_{1}$ :

$$
h=B \sqrt{1 / t}, \quad B=C_{1} \sqrt{v x / g},
$$

where $B$ is a multiplicative factor for the temporal evolution, $x$ is a fixed position on the sample, and $v \equiv$ $(\mu / \rho)$ is the kinematic viscosity and where $C_{1}=f\left(C_{a}\right)$, i.e., a function of capillarity number $C a \equiv \mu U / \sigma .{ }^{3,12}$

\section{Results and Discussion}

Experiments were performed with three Newtonian, nonvolatile mineral oils with distinct viscosities and at three withdrawing speeds. An interferogram obtained from the temporal evolution of mineral oil OP60 is illustrated in Fig. 2, where each interval between successive extremes corresponds to an optical thickness (physical thickness multiplied by refractive index) variation of $\lambda / 4$.

Through these $\lambda / 4$ step intervals, Figs. 3 and 4 follow. In Fig. 3 the evolution of the optical thickness of oil OP60 is shown for three withdrawing 


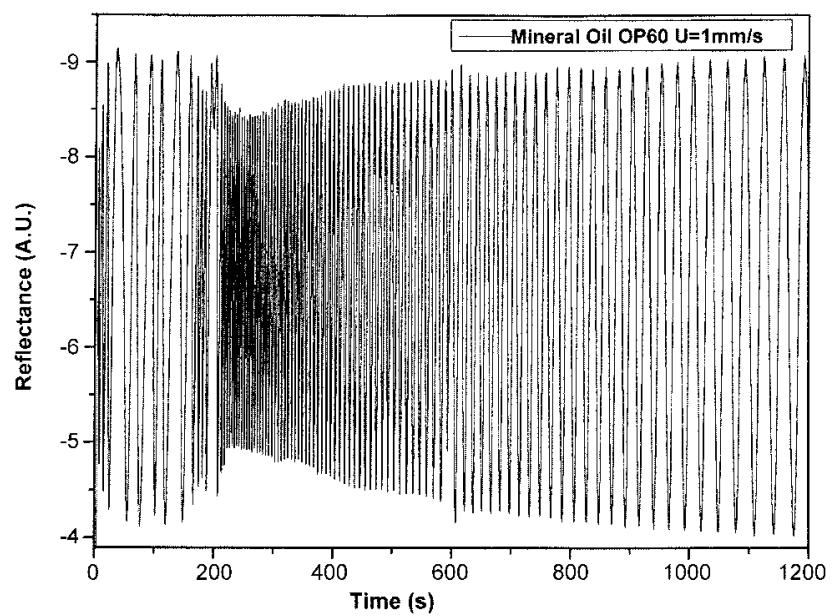

Fig. 2. Temporal evolution of reflectance for mineral oil OP60 during dip coating. Refractive indices $(n)$ at $660 \mathrm{~nm}: 1.503 \pm$ 0.001 for a glass substrate (Brewster method) and $1.470 \pm 0.001$ for mineral oil OP60 (Abbe refractometry).

speeds. The same speeds for oils OP20, OP60, and OP400 are shown in Fig. 4.

From Fig. 3 we note that, as the withdrawing speed increases, so does the mass drain in the same time interval. Equation (10), however, indicates that a thicker film adheres to the substrate for a faster withdrawing speed. These two observations are consistent, as a thicker film permits a faster flow in its outermost layers.

Similarly, we note from Fig. 4 that, as the oil's viscosity increases (see Table 1), so does the mass loss in the same time interval. This does not imply a smaller optical thickness value, which would contradict Eq. (10), because the initial thickness is greater for higher viscosity.

We also verify that the experimental data, within an uncertainty of $\pm 7 \mathrm{~nm}$, agree with values obtained from Eq. (10), with the exception of the initial stage of the process. After this stage, which corresponds to

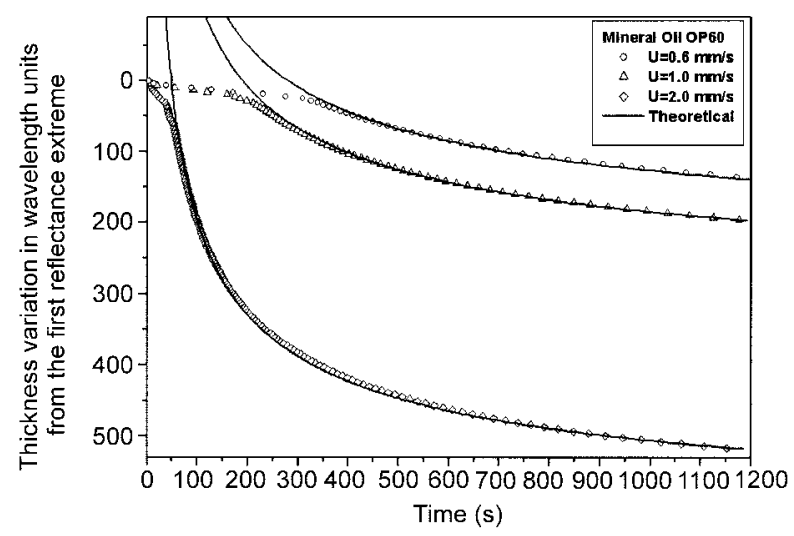

Fig. 3. Thickness variation, in wavelength units, for a film of mineral oil OP60 during dip coating at several withdrawing speeds $(U)$. Although experimental points were obtained at each $\lambda / 4$ variation, for clarity we show only data at each $\lambda$ variation. The experimental uncertainty is of the order of $\lambda / 50$.

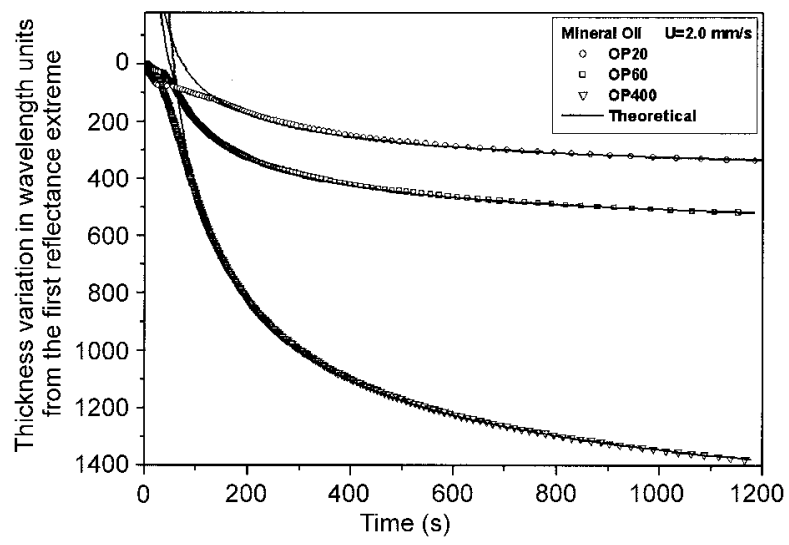

Fig. 4. Thickness variations in wavelength units, for films of mineral oils OP20, OP60, and OP400 during dip coating at the same withdrawal speed $(U)$. Although experimental points were obtained at each $\lambda / 4$ variation, for clarity we show only data at each $\lambda$ variation. The experimental uncertainty is of the order of $\lambda / 50$.

Table 1. Physical Properties of the Oils under Analysis during Dip Coating $^{a}$

\begin{tabular}{ccc}
\hline \multicolumn{3}{c}{ Kinematic Viscosity } \\
Oil & $\left(v=\mu / \rho ; \mathrm{mm}^{2} / \mathrm{s}\right)$ & Refractive Index \\
\hline OP20 & 36 & $1.461+-0.001$ \\
OP60 & 116 & $1.470+-0.001$ \\
OP400 & 1055 & $1.492+-0.001$ \\
\hline
\end{tabular}

${ }^{a}$ The kinematic viscosity values were provided by the manufacturer, and refractive indices were determined by Abbe refractometry.

an asymptotic behavior in Eq. (10), the agreement corroborates the theoretical model with fitting parameter $C_{1}$, compatible with previous reports that involve other Newtonian, nonvolatile liquids..$^{1,3}$

Furthermore, as was previously assumed by White and Tallmadge, ${ }^{12}$ the agreement between theoretical predictions and measured results confirms that the continuous dip-coating model can be applied with the continuity equation to the batch process under steady-state conditions, and the edge effects disregarded.

\section{Concluding Remarks}

We have presented what is to our knowledge the first optical monitoring analysis during batch dip coating of mineral oils. With a refractometry measurement and an acquisition rate of $3.5 \mathrm{kHz}$, a quantitative description of the temporal variation in thickness was achieved from the beginning of the process.

After the initial stage, the experimental data agreed well with the $t^{-1 / 2}$ dependence predicted by a simple theoretical model, with a fitting parameter related to the capillarity number.

The precision of the dip-coating process is 2 orders of magnitude better than that attained by traditional capacitive measurements. As a consequence, the method permits the characterization of interferomet- 
ric, liquid layer thicknesses with nanometric precision, thus opening new perspectives for analysis of the dynamics of dip coating.

We are grateful to the Instituto de Pesquisas Tecnológicas for providing the oil standards. This research was partially supported by the Program de Apoio ao Desenvolvimento Científico e Tecnológio, Conselho Nacional de Desenvolvimento Científico e Technológico, and the Coordenação de Aperfieçoamento de Pessoal de Nível Superior (Brazil).

\section{References}

1. L. E. Scriven, "Physics and applications of dip coating and spin coating," Mater. Res. Soc. Symp. Proc. 121, 717-729 (1988).

2. P. R. Schunk, A. J. Hurd, and C. J. Brinker, "Free-meniscus coating processes," in Liquid Film Coating, S. F. Kistler and P. M. Schweizer, eds. (Chapman \& Hall, London, 1997), Chap. 13.

3. R. P. Spiers, C. V. Subabaraman, and W. L. Wilkinson, "Free coating of a Newtonian liquid onto a vertical surface," Chem. Eng. Sci. 29, 389-396 (1973).

4. H. A. Macleod, Thin-Film Optical Filters, 2nd ed. (Macmillan, New York, 1986), p. 424
5. F. Horowitz, E. M. Yeatman, E. Dawnay, and A. Fardad, "Optics as a key to spin coating sol-gel films," in 16th Congress of the International Commission for Optics: Optics as a Key to High Technology, G. Akos, T. Lippenyi, G. Lupkovics, and A. Podmaniczky, eds., Proc. SPIE 1983, 1022-1023 (1993).

6. F. Horowitz, E. M. Yeatman, E. Dawnay, A. Fardad, and Mino Green, "Real-time optical monitoring of spin coating," J. Phys. III 3, 2059-2063 (1993).

7. D. Qu, E. Rancé, and S. Garoff, "Dip coated films of volatile liquids," Phys. Fluids 14, 1154-1156 (2002).

8. F. Horowitz, A. F. Michels, P. Alcantara, Jr., M. B. Pereira, A. P. Rizzato, and C. V. Santilli, "Real-time interferometric monitoring of dip coating," in 18th Congress of the International Commission for Optics, A. J. Glass, J. W. Goodman, M. Chang, A. H. Guenther, and Toshimitsu Asakura, eds., Proc. SPIE 3749, 729-730 (1999).

9. L. D. Landau and B. G. Levich, "Dragging of a liquid by a moving plate," Acta Physicochim. URSS 17, 42-54 (1942).

10. B. G. Levich, Physicochemical Hydrodynamics (Prentice-Hall, Englewood Cliffs, N.J., 1962), Chap. 12.

11. C. Gutfinger and J. A. Tallmadge, "Films of non-Newtonian fluids adhering to flat plates," AIChE. J. 11, 403-413 (1965).

12. D. A. White and J. A. Tallmadge, "Theory of drag out of liquids on flat plates," Chem. Eng. Sci. 20, 33-37 (1965). 